Artículo Original. Septiembre-Diciembre 2017; 7(3):72-82. Recibido: 15/05/2017 Aceptado: 20/07/2017.

http://dx.doi.org/10.21929/abavet2017.73.8

\title{
Presencia de estructuras sugestivas de Ehrlichiosis en perros de la ciudad de Tepic Nayarit.
}

A presence of structures suggestive of Ehrlichiosis in dogs from Tepic city Nayarit.

\author{
González-Morteo Carlos cgmorteo@uan.edu.mx, De la Cruz-Moreno Omar \\ rowland_jonas@hotmail.com, Álvarez-Guerrero Cesar uniaun@hotmail.com, Borrayo- \\ González Juan mvz_borrayo1@hotmail.com
}

Unidad Académica de Medicina Veterinaria y Zootecnia, Universidad Autónoma de Nayarit. Nayarit. México. Autor responsable y de correspondencia González-Morteo Carlos. Carretera ChapalillaCompostela, km 3.5, Compostela Nayarit. México.

\section{RESUMEN}

Se realizó un estudio epidemiológico en 6 clínicas de Tepic Nayarit, con el propósito de cuantificar la presencia de Ehrlichia spp., en la sangre periférica de perros sospechosos de este padecimiento. En un periodo de 9 meses se pudieron observar 389 muestras, de ellas 210 (53.98\%) de hembras, y 179 (46.02\%) de machos. Las muestras fueron teñidas con colorante de Wright. Se observó minuciosamente al microscopio, en busca de mórulas características de la enfermedad. Resultaron 45 hembras positivas y 6 machos positivos, se hizo una prueba de ANDEVA que resultó altamente significativa $(P<0.05)$ para la diferencia entre sexos. La prevalencia por sexo fue de $21.42 \%$ en hembras y $3.35 \%$ para machos. Prevalencia integral fue de 13.11. Se concluye que la enfermedad es un factor de riesgo para la salud pública del Estado de Nayarit, ya que este reúne todas las características medio ambiente favorables para el desarrollo de esta enfermedad zoonótica.

Palabras clave: bacteria, zoonosis, diagnóstico, prevalencia.

\begin{abstract}
In six veterinary clinics of Tepic Nayarit, it was made an epidemiological study about the presence of Ehrlichia spp. The blood of suspects dogs was used. In 9 months there were 389 blood samples. 210 from females and 179 from males dogs. All samples were stained with Wright and microscopically carefully observed, looking for characteristic morulas. It was Found 45 females and 6 males positives. In ANDEVA statistical analysis was higher significance $(P<0.05)$ between sexes. Prevalence by sex was $21.42 \%$ females and $3.35 \%$ males. The total Prevalence was $13.11 \%$. it is Conclude this disease is high risk for public health in Nayarit state because environmental conditions are optimum for Ehrlichiosis zoonosis.
\end{abstract}

Key words: bacterium, zoonosis, diagnosis, prevalence.

\section{INTRODUCCIÓN}

Las Rickettsiosis son enfermedades causadas por bacterias del género Rickettsia; constituyen un grave problema sanitario y de gran impacto a nivel mundial, ante la posibilidad de afectar amplios sectores de la población y la dificultad en el diagnóstico; los desenlaces suelen ser fatales cuando no son detectados de forma temprana (Buitrago y Pachón, 2008). Las Rickettsias son Gram negativas intracelulares 
obligadas, pertenecientes a la familia Rickettsiaceae. Se clasifican en tres grupos de acuerdo con sus características genéticas: grupo ancestral compuesto por $R$. bellii y $R$. canadensis, grupo tifus compuesto por R. prowasekii y R. typhi, y grupo de las fiebres manchadas. Este último grupo incluye más de veinte especies transmitidas por garrapatas, entre ellas están: $R$. rickettsii, $R$. conorii, $R$. sibirica, $R$. japonica y dos especies transmitidas por vectores distintos de garrapatas, que son la $R$. akari, transmitida por un ácaro del ratón Liponyssoides sanguineus y $R$. felis por pulgas (López et al., 2007). Se ha descubierto que la Rickettsia spp. en perros, muestra una seroprevalencia del $26 \%-60 \%$ en regiones endémicas (Solano et al., 2006).

Es importante tomar en cuenta que existen enfermedades que se pudieran considerar con nula presencia o que simplemente están siendo subdiagnosticadas, entre ellas nos encontramos con enfermedades de muy diversa índole: 1) las de origen alimentario, 2) las que se transmiten por contacto con animales domésticos o salvajes, 3) las que se asocian con estados inmunodepresivos y 4) las zoonóticas que son transmitidas por vectores (EMERGING, 2002).

Las Ehrlichiosis son enfermedades muy conocidas en la medicina veterinaria desde hace varias décadas, sin embargo, su estudio en humanos es reciente. La mortalidad es escasa (2-3\%), pero puede ocurrir como resultado de complicaciones con otras infecciones (Rojas y Villalobos, 2007; Rey et al., 2009).

Las Ehrlichias se agrupan de acuerdo al tipo de célula de sangre que más comúnmente infectan, como son los granulocitos, linfocitos, monocitos y plaquetas. Las enfermedades derivadas de estas afecciones fueron nombradas en consecuencia. Varias de estas especies de la familia Anaplasmataceae infectan a los caninos, incluyendo las especies Ehrlichia canis, Ehrlichia chaffeensis y Ehrlichia ewingii; siendo Ehrlichia Chaffeensis, una especie estrechamente relacionada con E. canis, trasmitida por Rhipicephalus sanguineus (Heyman et al., 2007).

Las Ehrlichias son de crecimiento intracelular obligado tanto en los leucocitos animales como en los humanos; crecen en los fagosomas de las células infectadas, evaden las defensas, inhibiendo la fusión de los fagosomas con los lisosomas. Las microcolonias de Ehrlichias que se forman en una vacuola intra-citoplasmática, adquiere el nombre de mórula (Barcat, 2006). Durante el 2001, el género Ehrlichia junto con otros géneros relacionados como lo son el Cowdria, Neorickettsia y Wolbachia se reorganizaron y se incluyeron en la familia Anaplasmataceae; Quedando reagrupados los géneros Ehrlichia platys, Ehrlichia bovis y las especies de Ehrlichia phagocitophila, además de incluir el género Anaplasma. Observándose como característica principal en Anaplasma platys la capacidad de infectar plaquetas en sangre de los perros, células que en su interior muestran mórulas con una cantidad variable de inclusiones (Aguirre et al., 2006). 
La Ehrlichiosis canina es una enfermedad infecciosa e inmunodepresiva, que se trasmite por el vector Rhipicephalus sanguineus conocida como la garrapata marrón del perro, infectando a caninos domésticos y silvestres. Es también llamada "enfermedad del perro rastreador", "pancitopenia canina tropical", "fiebre canina hemorrágica', y “tifus canina” (López et al., 1999; Adrianzén et al., 2003).

Desde hace varios años se ha incrementado el número de casos con Ehrlichiosis Canina en diversas áreas geográficas del mundo, clasificándola como una enfermedad emergente (Stafford, 2004; Hoyos et al., 2007). La Ehrlichia canis y Ehrlichia chaffeensis son responsables de la Ehrlichiosis monocítica canina y Ehrlichiosis monocítica humana respectivamente; Sin embargo, ambas bacterias han sido reportadas tanto en perros como en humanos; infectando principalmente células sanguíneas, en particular células mononucleares multiplicándose dentro de vacuolas intracitoplasmáticas, hasta formar las inclusiones basófilas características llamadas mórulas (Gutiérrez et al., 2009).

La Ehrlichiosis monocítica canina es endémica en la región oeste de Turquía, región en la cual existe una seroprevalencia del $21 \%$; esta seroprevalencia varía y depende de la ubicación y la población de los perros. El diagnóstico clínico puede ser confirmado mediante la demostración de organismos intracitoplasmásticos, llamados mórula dentro de los glóbulos blancos. Con mayor frecuencia el diagnóstico se realiza mediante una combinación de signos clínicos, la presencia de mórulas en los leucocitos, títulos positivos, fluorescencia indirecta de anticuerpos séricos y la respuesta positiva al tratamiento (Icen et al., 2010).

Por otra parte, Ehrlichia Ewinggi es el agente causante de la Ehrlichiosis canina granulocítica. Estudios experimentales sugieren que la transmisión de ésta puede ser transestadial, transmitida por la garrapata del género Amblyomma Americanum. Además de las especies ya conocidas de Ehrlichia que afectan a los caninos, existen otros dos agentes que se consideran importantes patógenos caninos como los son, Anaplasma phagocitophilum y Anaplasma platys, infectando este último las plaquetas del perro, provoca una Trombocitopenia Cíclica Canina (CCT) (Heyman et al., 2007).

El Amblyoma Americanun es el principal vector del agente patógeno Ehrlichia Ewingii, al igual que para $E$. Chaffeensis; infecta este último con más frecuencia los monocitos, mientras que Phagocytophilum Ewingii, muestra mayor predilección por los granulocitos. La Ehrlichiosis y Anaplasmosis se caracterizan por la infección de leucocitos, en donde los agentes causantes se multiplican en la membrana citoplasmática (Chapman et al., 2006). 
La mayor incidencia de ehrlichiosis se produce en los meses en los que las diferentes especies de garrapatas implicadas en la transmisión de estas bacterias están más activas (primavera-verano y principio de otoño) (Anaya et al., 2009).

En Nayarit se ha observado un incremento de perros con los signos de Ehrlichiosis, sin embargo, como en todas las enfermedades emergentes, el problema del diagnóstico certero y oportuno aún no se resuelve, por el hecho que los kits de diagnóstico se tienen que importar y esto resulta caro para el clínico y/o el propietario del perro; entonces se tratan los pacientes con los fármacos que recomienda la literatura y el clínico determina que el caso que tuvo se debía a la Ehrlichia.

\section{MATERIAL Y MÉTODOS}

El estudio se realizó en la ciudad de Tepic, capital del Estado de Nayarit; la cual se ubica en las coordenadas geográficas extremas en una longitud oeste $104^{\circ}, 5^{\prime}$ y $105^{\circ}$ $05^{\prime}$ y una latitud Norte $21^{\circ}, 3^{\prime}$, a $21^{\circ}, 6^{\prime}$ y una altura promedio de 900 mts. s.n.m. Predominan dos tipos de clima; el cálido subhúmedo con lluvias en verano y el semicálido subhúmedo con lluvias en verano; la precipitación promedio anual es de $1.121 \mathrm{~mm}$; se reporta una temperatura promedio de $21.1^{\circ} \mathrm{C}$ (INEGI, 2015).

El muestreo se hizo en el periodo de enero a septiembre 2015, en 6 clínicas veterinarias de la ciudad de Tepic, con un total de 389 animales muestreados, con cuadro clínico sospechoso de Ehrlichiosis.

El propósito fue delimitar la búsqueda de aquellos perros que presentaban algunos signos de la enfermedad (fiebre con depresión, anorexia, pérdida de peso, petequias o hemorragia retinal, ataxia, disfunción vestibular; así como signos respiratorios como disnea y aumento de sonidos broncovesiculares). Se recolectó sangre (2-3 ml) a partir de la vena radial de los perros, utilizando tubos con anticoagulante (EDTA), las muestras se conservaron en refrigeración $\left(4^{\circ} \mathrm{C}\right)$ previo al análisis.

En el laboratorio se realizó el frotis sanguíneo y se procedió a realizar la tinción de Wright; después se secó y se llevó a cabo la observación minuciosa para buscar las mórulas características de la enfermedad. Se observó al microscopio óptico de trasluminación con objetivos de 10X, 40X y 100X con aceite de inmersión (Weiss y Jane, 2010). 


\section{RESULTADOS}

En la revisión de los frotis teñidos se encontraron las mórulas características de la infección por Ehrlichia spp. Figuras 1 y 2.

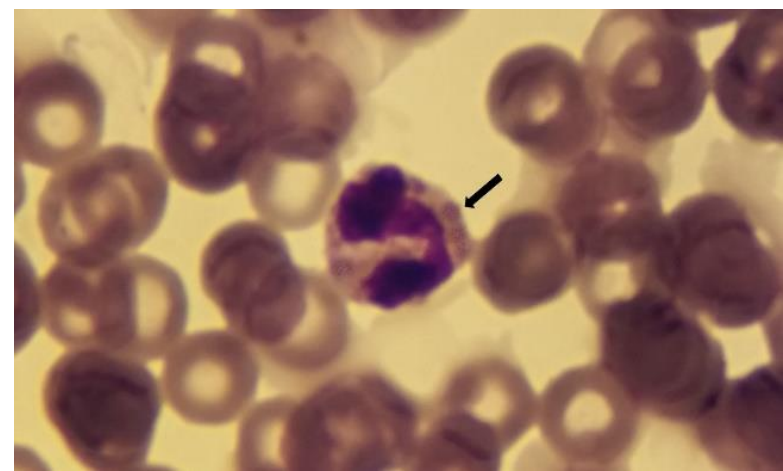

Figura 1. Frotis sanguíneo 1000x, en él se ve un glóbulo blanco con mórulas de la bacteria dentro del citoplasma (flecha).

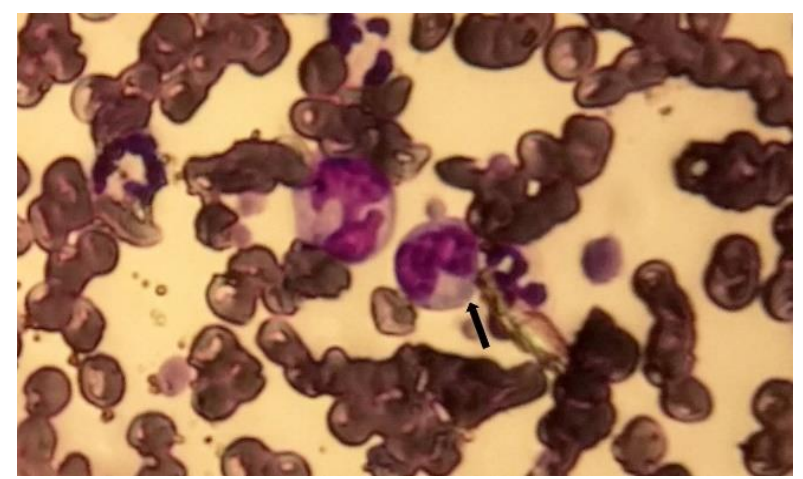

Figura 2. Frotis sanguíneo $800 x$, en él se observa glóbulos blancos con mórulas dentro del citoplasma (flecha).

\begin{tabular}{|c|c|c|c|c|c|c|}
\hline Clínica & Muestras & Sospech. & Pos. & Neg. & Hembras (+) & Machos (+) \\
\hline A & 64 & 6 & 15 & 43 & 14 & 1 \\
\hline B & 65 & 2 & 2 & 61 & 2 & 0 \\
\hline C & 64 & 7 & 4 & 53 & 2 & 2 \\
\hline D & 68 & 5 & 10 & 53 & 10 & 0 \\
\hline E & 64 & 5 & 12 & 47 & 12 & 0 \\
\hline $\mathbf{F}$ & 64 & 2 & 8 & 54 & 5 & 3 \\
\hline Totales & $\begin{array}{c}389 \\
\left(179{ }^{-} y\right. \\
210\end{array}$ & 27 & 51 & 311 & 45 & 6 \\
\hline
\end{tabular}

Cuadro 1. Muestra los resultados en números reales. Hembras y machos positivos

En la presentación de los resultados en los cuadros 1 y 2, se puede observar que en primer lugar, hay una diferencia importante entre las clínicas, en cuanto a los positivos; esto pudiera ser debido al sitio geográfico de la ciudad en donde se encuentra localizada la clínica; es decir por el pequeño microambiente y clima que por condiciones propiciadas por los mismos dueños de éstas, así como la cultura y economía en la atención responsable de las mismas, influya o favorezca la presencia e incidencia de estos vectores biológicos. 
Las mayores incidencias de casos sugerentes a positivos se observaron en cachorros de hasta 6 meses y en animales de un uno hasta cuatro años de edad. Del total de la muestra estudiada se presentaron 39 casos sugerentes a positivos en animales de raza y 12 perros positivos, mestizos.

\begin{tabular}{|c|c|c|c|c|c|c|}
\hline $\begin{array}{l}\text { Clíni } \\
\text { ca }\end{array}$ & $\begin{array}{c}\text { Muestras } \\
\text { n. }\end{array}$ & $\begin{array}{c}\text { Sospecho } \\
\text { sos } \%\end{array}$ & $\begin{array}{c}\text { Positivos } \\
\%\end{array}$ & $\begin{array}{c}\text { Negativos } \\
\%\end{array}$ & $\begin{array}{c}\text { Hembras } \\
\text { (+) \% }\end{array}$ & $\begin{array}{c}\text { Machos } \\
(+) \%\end{array}$ \\
\hline$A$ & 64 & $9.375 \%$ & $23.437 \%$ & $67.187 \%$ & $21.875 \%$ & $1.562 \%$ \\
\hline B & 65 & $3.076 \%$ & $3.076 \%$ & $93.846 \%$ & $3.076 \%$ & $0 \%$ \\
\hline C & 64 & $10.937 \%$ & $6.25 \%$ & $82.812 \%$ & $3.125 \%$ & $3.125 \%$ \\
\hline D & 68 & $7.352 \%$ & $14.705 \%$ & $77.941 \%$ & $14.705 \%$ & $0 \%$ \\
\hline$E$ & 64 & $7.812 \%$ & $18.75 \%$ & $73.437 \%$ & $18.75 \%$ & $0 \%$ \\
\hline $\mathbf{F}$ & 64 & $3.125 \%$ & $12.5 \%$ & $84.375 \%$ & $7.812 \%$ & $4.687 \%$ \\
\hline Total & $46.01 \%$ & & & & $21.42 \%$ & $3.35 \%$ \\
\hline es & $+53.98 \%$ & & & & & \\
\hline
\end{tabular}

Cuadro 2. Resultados expresados en porcentajes. Los porcentajes de positivos en hembras y machos son con respecto a los machos y hembras muestreados.

Hubo 27 animales del total de la muestra que se consideraron como sospechosos, debido a que estos presentaron de una a dos mórulas dentro del citoplasma de los glóbulos blancos; éstas no se consideran suficientes para poder dar un diagnóstico positivo, o bien la imagen de éstas no fue convincente.

En el análisis de positivos entre hembras y machos se encontró una gran diferencia numérica, por lo tanto, se analizaron los datos con un programa de estadística SAS, con la prueba de ANDEVA; con lo que se estableció que la diferencia entre hembras y machos es estadísticamente significativa $(P<0.05)$.

En suma, se encontró una prevalencia total de $13.11 \%$ (de $3.35 \%$ en los machos y de $21.42 \%$ en las hembras).

\section{DISCUSIÓN}

Llama mucho la atención el haber encontrado una diferencia entre hembras positivas y machos positivos, se encontró que ésta es significativa $(P<0.05)$. Las hembras presentaron más casos positivos, se supone que pudiera ser tal vez que el desgaste físico que representa el embarazo, el parto y la lactancia las haga más susceptibles a la 
enfermedad y a las garrapatas; sin embargo, esta hipótesis con los resultados obtenidos no se puede corroborar; no obstante, Adrianzén et al., (2003), señala una situación semejante a nuestros resultados. En los frotis estudiados se pudo observar la presencia de plaquetas dañadas, según lo menciona Aguirre et al., (2006), para el caso de Anaplasma Platys, se consideró un diagnóstico; positivo a todo aquel frotis en el cual se pudo observar la presencia de mórulas dentro del citoplasma de los leucocitos y/o en las plaquetas.

Hoy en día las garrapatas se han convertido en uno de los principales vectores trasmisores de enfermedades infecciosas a nivel mundial (Johan et al., 2006); se les puede localizar en cualquier tipo de ecosistema; de ahí la importancia de tener un buen control de este parásito por lo menos en el lugar donde vive el perro.

En el presente estudio no se consideró la diferencia que existió en el manejo de acuerdo a la aplicación de baños garrapaticidas entre hembras y machos. En este orden de ideas, es importante enfatizar el buen manejo ambiental y de la mascota con productos que permitan el romper el ciclo biológico del vector.

La presencia de Ehrlichiosis queda evidenciada en los casos con signos clínicos y/o antecedentes de exposición a garrapatas; el examen de laboratorio es de gran utilidad para el diagnóstico. Icen et al. (2010).

De los resultados obtenidos en el presente trabajo, se demuestra que por los 51 casos con signos y daño encontrado en las mórulas características de la infección por Ehrlichia spp, tiene una gran relevancia la convivencia con perros portadores Ehrlichia Canis en hogares; siendo un factor que llega a determinar la adquisición de la enfermedad en humanos, ya que es probable que este vector sea el agente trasmisor de esta zoonosis animal-humano. Se considera que algunos factores como la garrapata marrón del perro, muerde ocasionalmente al humano; a diferencia de algunas especies como Amblyoma spp. (Abarca et al., 2008). De tal forma se considera tanto en la Medicina Veterinaria como en la Medicina humana, un problema de salud pública en nuestro país.

La Ehrlichia spp puede ser transmitida por una gran variedad de hospedadores naturales, pero en muchos lugares se desconoce el rol potencial del perro como un reservorio zoonótico, convirtiéndose en un factor de riesgo para humanos (Benavidez y Ramírez, 2003). Es importante realizar pruebas de laboratorio más específicas que nos ayuden a determinar con mejor exactitud, la presencia de anticuerpos de Ehrlichia en animales con signos clínicos que indique la sospecha de esta enfermedad; y en un futuro poder diferenciar las diversas especies de Ehrlichia y/o otras Rikettsias presentes en la población canina municipal, estatal y nacional. 
La Ehrlichiosis Canina representa un foco importante de infección activa, tanto para las especies animales, como para los habitantes de la ciudad de Tepic, Nayarit. Los perros que permanecen más horas del día en la calle, tienen mayor contacto con el medio ambiente, lugar donde las garrapatas vectores realizan la muda entre sus estadíos (Adrianzén et al., 2003); por lo tanto se convierten en animales con mayor número de posibilidades de infectarse y por consecuencia de poder infectar el hogar en donde viven; siendo las manifestaciones hemorrágicas con presencia o no de fiebre, los principales signos clínicos de alerta para el diagnóstico presuntivo de la enfermedad.

La trombocitopenia y la leucopenia fueron las alteraciones hematológicas de mayor significación en los animales clínicamente enfermos.

Los dueños son el grupo de personas con mayor riesgo, lo cual se debe al contacto diario y permanente con los animales; en segundo lugar, se encuentran todos aquellos que prestan algún servicio profesional, como puede ser la estética, adiestramiento, cuidado, exposición o de consulta médica. Debido a que cada día la globalización es más frecuente en nuestras vidas, debemos prestar mayor atención al momento de querer importar o exportar animales sanos en donde la enfermedad es endémica.

En nuestro país existen pocos estudios acerca de la Ehrlichiosis spp, de sus vectores y de sus hospederos; de ahí la relevancia de continuar con este tipo de estudios y ampliarlos hacia los animales, con el fin de analizar de mejor forma la trasmisión de esta zoonosis (Anaya et al., 2009); de igual modo implementar medidas de prevención, por lo que tener una prueba diagnóstica es útil para la Ehrlichiosis spp., así como el conocimiento de su presencia en el país, contribuirá a una mejor vigilancia de la enfermedad

\section{CONCLUSIÓN}

La prevalencia total encontrada de esta enfermedad fue de $13.11 \%$ en el periodo de enero a septiembre del año 2015. La prevalencia en machos fue menor estadísticamente significativa $(P<0.05)$ con relación a las hembras $3.35 \%$ y $21.42 \%$ respectivamente. Todos los perros muestreados se encontraron sin signos clínicos.

\section{LITERATURA CITADA}

ABARCA VK, López Del PJ, González AP, Dabanch PJ, Torres HM, Solari GV, Perret PC. Evidencia seroepidemiológica de exposición humana a Anaplasma sp en Santiago, Chile. Pontificia Universidad Católica de Chile Santiago de Chile. Rev Chil Infect. 2008; 
25 (5): 358-361. ISSN: 0716/1018, DOI:10.4067/S0716-10182008000500008. http://www.scielo.cl/scielo.php?script=sci arttext\&pid=S0716-10182008000500008

ADRIANZÉN GJ, Chávez VA, Casas AE, Li EO. Seroprevalencia de la Dirofilariosis y Ehrlichiosis canina en tres distritos de lima. Rev Inv Vet Perú. 2003. 14 (1):43-48._ISSN: 1609-9117.

http://revistasinvestigacion.unmsm.edu.pe/index.php/veterinaria/article/view/1596

AGUIRRE E, Tesouro MA, Ruiz L, Amusategui I, Sainz A. Genetic Characterization of Anaplasma (Ehrlichia) platys in Dogs in Spain. J. Vet. Med. 2006; 53:197-200. PMID: 16629989 DOI: $10.1111 /$ j.1439-0450.2006.00937.x

https://www.ncbi.nlm.nih.gov/pubmed/16629989

ANAYA E, Morón C, Jaramillo K, Mendoza L, Román R. Evidencia serológica de Ehrlichiosis Humana en Ancash, Perú. Rev Peru Med Exp Salud Pública. 2009; 26(1):5457. ISSN: 1726-4634

http://www.academia.edu/4027873/EVIDENCIA SEROL\%C3\%93GICA DE EHRLICHI OSIS HUMANA EN ANCASH PER

BARCAT JA. El calentamiento global, las garrapatas y la Ehrlichiosis. Medicina (Buenos $\begin{array}{lllll}\text { Aires). } & 2006 ; & 66 & (5): 489-491 . & \text { ISSN: }\end{array}$ http://www.scielo.org.ar/scielo.php?script=sci arttext\&pid=S0025-76802006000500020

BENAVIDES JA, Ramírez JF. Ehrlichiosis canina, casos clínicos. Rev. Col. Cienc. Pec. 2003; 16 (3):268-274. https://dialnet.unirioja.es/descarga/articulo/3241943.pdf

CHAPMAN AS, Bakken JS, Folk SM, Paddock CD, Bloch KC, Krusell A, Sexton DJ, Buckingham SC, Marshall GS, Storch GA, Dasch GA, McQuiston JH, Swerdlow DL, Dumler JS, Nicholson WL, Walker DH, Eremeeva ME. Ohl CA. A Practical Guide for Physicians and Other Health-Care and Public Health Professionals Diagnosis and Management of Tickborne Rickettsial Diseases: Rocky Mountain Spotted Fever, Ehrlichioses, and Anaplasmosis United States. 2006; 55(RR-4):1-27. https://www.cdc.gov/mmwr/preview/mmwrhtml/rr5504a1.htm

EMERGING bacterial zoonoses. Rev Panam Salud Publica [online]. 2002;11(1):50-55. ISSN 1680-5348. $\quad$ http://dx.doi.org/10.1590/S1020-49892002000100017. http://www.scielosp.org/scielo.php?script=sci abstract\&pid=S1020$49892002000100017 \& \operatorname{lng}=$ en\&nrm=iso\&tlng=en

GUTIÉRREZ GCN, Martínez AM C, Triana-Alonso FJ. Identificación microscópica y molecular de ehrlichias en perros del estado Aragua-Venezuela. Salus online. 2009; 12 (Sup. 1) Biología Molecular - Ehrlichias en perros 197. http://www.scielo.org.pe/scielo.php?pid=S1609-91172015000400012\&script=sci arttext\&tlng=en 
HEYMAN P, Duh D, Van Der Kuylen B, Cochez C, Van Esbroeck M, Vandenvelde C, Avsic-Zupanc T. Molecular and Serological Evidence for Anaplasma platys and Babesia sp. Infection in a Dog, Imported in Belgium, from Southern Spain. J. Vet. Med. A. 2007; 54: 276-279. PMID: 17523964 DOI: 10.1111/j.1439-0442.2007.00872.x https://www.ncbi.nlm.nih.gov/pubmed/17523964

HOYOS SL, Li EO, Alvarado SA, Suárez AF, Díaz CD. Evaluación del examen hematológico en el diagnóstico de Ehrlichiosis Canina. Rev Inv Vet Perú. 2007; 18 (2):129-135.

http://revistasinvestigacion.unmsm.edu.pe/index.php/veterinaria/article/view/1288

Icen H, Sekin S, Simsek A, Kochan A, Celik OY, Altas MG. Prevalence of Dirofilaria immitis, Ehrlichia canis, Borrelia burgdorferi infection in dog from Diyarbakir in Turkey. Asian Journal Of Animal And Veterinary Advances. 2010; 6 (4): 371-378. ISSN: 16839919, DOI: 10.3923/ajava.2011.371.378. http://scialert.net/fulltext/?doi=ajava.2011.371.378\&org=10

Instituto Nacional de Estadística y Geografía disponible en red en: http://mapserver.inegi.org. $\mathrm{mx} / \mathrm{mgn} 2 \mathrm{k} / \mathrm{?s}=\mathrm{geo \& c=954}$. Consultado el 04 de Noviembre de 2015 a las 11:28 A. M.

Stafford III KC. An integrated guide for homeowners, pest control operators, and public health officials for the prevention of tick-associated disease Tick Management Handbook The Connecticut Agricultural Experiment Station. New Jersey. USA. 2004:1-71. http://www.ct.gov/caes/lib/caes/documents/special features/tickhandbook.pdf

LÓPEZ DPJ, Abarca V K, Azócar AT. Evidencia clínica y serológica de rickettsiosis canina en Chile. Rev chil infect. 2007; 24:189-193, ISSN: 0716-1018. DOI:10.4067/S071610182007000300002. http://www.scielo.cl/pdf/rci/v24n3/art02.pdf

LÓPEZ J, Castillo A, Muñoz M, Hildebrandt S. Hallazgo de Ehrlichia canis en Chile, informe preliminar. Arch. Med. Vet. 1999; 31(2): 211-214, ISSN: 0301-732X, DOI: 10.4067/S0301-732X1999000200008.

http://www.scielo.cl/scielo.php?script=sci arttext\&pid=S0301-732X1999000200008

CARRILLO BLM, Betancur CS, Roldán CD, Pérez JJE, Galeano RD, Loaiza EET, Giraldo ECA. Implementación de un método basado en PCR, para diagnóstico de Ehrlichia spp., en caninos de Medellín (Colombia). Ces. Med. Vet. Zootec. 2012; 7(2). ISSN: 1900-9607.

REY JR, Lord CC, Connely RC. Ehrlichia y Anaplasma en Florida. Serie de publicaciones del Departamento de Entomología y Nematología del Servicio de Extensión Cooperativo de la Florida, del Instituto de Alimentos y Ciencias Agrícolas, Universidad de la Florida. 2002. http://edis.ifas.ufl.edu/in422 
ROJAS-SOLANO JR, Villalobos-Vindas J. Caso clínico. Ehrliquiosis granulocitotrópica Humana. Acta Médica Costarricense. 2007; 49 (2):121-123, ISSN 0001-6002. http://www.scielo.sa.cr/pdf/amc/v49n2/3460.pdf

SOLANO-GALLEGO L, Kidd L, Trotta M, Di Marco M, Caldin M, Furlanello T, Breitschwerdt E. Febrile Illness Associated with Rickettsia conorii Infection in Dogs from Sicily. Emerging Infectious Diseases. 2006; 12:1985-1988, PMCID: PMC3291343, DOI: 10.3201/eid1212.060326.

https://www.ncbi.nlm.nih.gov/pmc/articles/PMC3291343/pdf/06-0326.pdf

ULUTAS B, Bayramli, G, Karagenc T. First Case of Anaplasma (Ehrlichia) platys Infection in a Dog in Turkey. Department of Internal Medicine and Parasitology Faculty of Veterinary Medicine, Adnan Menderes University, Turkey, Turk. J. Vet. Anim. Sci. 2007; 31(4): 279282.

http://journals.tubitak.gov.tr/veterinary/abstract.htm;jsessionid=A98E1593E5380B1D324 E15D6F417F36A?id=9077

WEISS DJ, Wardrop KJ. Schalm's Veterinary Hematology, $6^{\text {th }}$ edition. Blackwell publishing. Ames lowa. 2010, ISBN: 978-0-8138-1798-9. http://www.armchairpatriot.com/HomeVet/Schalm's\%20Veterinary\%20Hematology\%206th\%20ed\%20\%20D.\%20Weiss,\%20J.\%20Wardrop\%20(Wiley-Blackwell,\%202010)\%20WW.pdf 